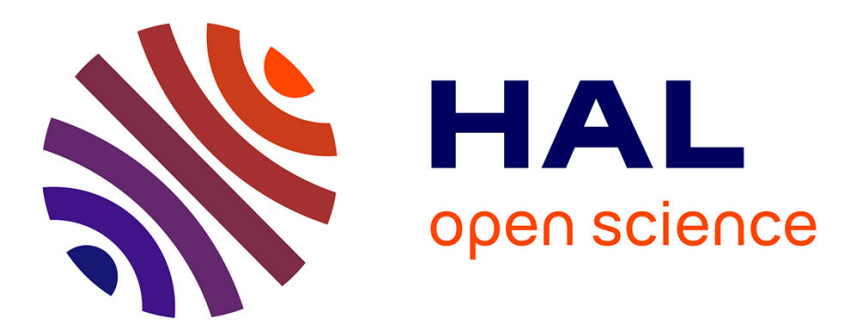

\title{
PROPAGATION GUIDÉE EN PRÉSENCE DE CHAMPS DE VITESSE ET DE TEMPÉRATURE STRATIFIÉS
}

\author{
Y. Gervais, J. Peube
}

\section{- To cite this version:}

Y. Gervais, J. Peube. PROPAGATION GUIDÉE EN PRÉSENCE DE CHAMPS DE VITESSE ET DE TEMPÉRATURE STRATIFIÉS. Journal de Physique IV Proceedings, 1992, 02 (C1), pp.C1-549C1-552. 10.1051/jp4:19921118 . jpa-00251073

\section{HAL Id: jpa-00251073 https://hal.science/jpa-00251073}

Submitted on 1 Jan 1992

HAL is a multi-disciplinary open access archive for the deposit and dissemination of scientific research documents, whether they are published or not. The documents may come from teaching and research institutions in France or abroad, or from public or private research centers.
L'archive ouverte pluridisciplinaire HAL, est destinée au dépôt et à la diffusion de documents scientifiques de niveau recherche, publiés ou non, émanant des établissements d'enseignement et de recherche français ou étrangers, des laboratoires publics ou privés. 


\title{
PROPAGATION GUIDÉE EN PRÉESENE DE CHAMPS DE VITESSE ET DE TEMPÉRATURE STRATIFIES
}

\author{
Y. GERVAIS et J.L. PEUBE \\ Laboratoire d'Etudes Aérodynamiques, 40 Avenue du Recteur Pineau, F-86022 Poitiers cedex, France
}

\begin{abstract}
We consider here the propagation of sound waves in a circular duct containing a non-uniforme non-isothermal laminar flow. The equation of propagation for the acoustic pressure in such a medium is given, and resolved considering downstream and upstream propagation of modes. We also give the analytical expression of the phase velocity of the longitudinal mode in these particular cases of propagation from a perturbation method.
\end{abstract}

\section{INTRODUCTION}

La présence d'un écoulement non-uniforme dans un guide d'onde a pour principal effet la réfraction des ondes dûe au cisaillement de vitesse moyenne. Ce phénomène, qui a fait l'objet de nombreuses études [1] [2] [3], reste à exanimer lorsque l'écoulement moyen est non-isotherme. En effet, la présence simultanée de champs de vitesse et de température moyennes non-uniformes perturbe notablement la propagation. Ces deux champs agissent en fait différamment, de telle sorte qu'il est bien souvent difficile en pratique d'en négliger un devant l'autre. On étudie ici la propagation établie des modes dans une telle configuration, en laissant de coté le problème des modes évanescents, ainsi que les phénomènes d'amortissement visqueux ou thermique.

\section{EQUATION D'ONDE EN MILIEU INHOMOGENE}

On considère la propagation d'ondes acoustiques dans une conduite cylindrique circulaire de longueur infinie et de paroi rigide, où règnent simultanément des champs de vitesse et de températures moyennes stratifiés. L'écoulement moyen stationnaire s'effectue selon l'axe $\mathbf{O} \vec{z}$ de la conduite et est indépendant de celui-ci, de telle sorte que les répartitions de vitesse et de température moyennes sont entièrement définies par les fonctions $\bar{U}(r, \theta)$ et $\bar{T}(r, \theta)$.

La propagation des ondes dans de telles conditions répond aux hypothèses habituelles de l'acoustique linéaire. Chaque grandeur peut donc être définie comme la somme d'une composante moyenne et d'une perturbation acoustique. Même si la présence d'un écoulement moyen non-uniforme est le fait de la viscosité, il est tout à fait légitime de négliger l'amortissement de l'onde lié à celle-ci, ou encore à la conduction thermique tant que les fréquences sont faibles. De plus, le fait que l'écoulement soit non-isotherme ne signifie pas pour autant que celui-ci soit générateur d'énergie acoustique. La propagation des ondes s'effectue donc de manière isentropique.

\section{Equation de propagation}

L'équation qui décrit la propagation acoustique en conduite cylindrique (définie par les variables d'espace $r, \theta, z$ ) en présence d'un écoulement non-isotherme s'obtient naturellement à partir des équations générales de la mécanique des fluides linéarisées, dans lesquelles on omet volontairement les termes liés à la viscosité (équations de conservation de la masse, du mouvement et de l'énergie). La relation d'énergie s'écrit ici : 


$$
\frac{\mathrm{dS}}{\mathrm{dt}}=0
$$

où $\mathrm{S}$ désigne l'entropie du fluide, somme d'une composante locale moyenne $\overline{\mathrm{S}}$ et de la perturbation acoustique $s$, et où d/dt symbolise la dérivée matérielle, définie par:

$$
\frac{d f}{d t}=\frac{\partial f}{\partial t}+\vec{U} \cdot \overrightarrow{g r a d} f
$$

Comme la relation d'état permet de définir la pression en fonction de la masse volumique et de l'entropie, on obtient, compte tenu de (1), la relation suivante entre les variations de pression et de masse volumique [4] :

$$
\frac{d \rho}{d t}=\frac{1}{c^{2}} \frac{d p}{d t}-\vec{U} \cdot \overrightarrow{\operatorname{grad}} \bar{\rho}
$$

où $\bar{\rho}$ désigne la valeur locale de la masse volumique.

A partir de la dérivée matérielle de l'équation de conservation de la masse, et des dérivées partielles des équations du mouvement dans les trois directions respectivement aux variables d'espace $r$, $\boldsymbol{\theta}$ et $\mathrm{z}$, on obtient l'équation de propagation des ondes acoustiques dans un milieu non-isotherme en mouvement :

$$
\Delta p-\frac{1}{c^{2}} \frac{\partial^{2} p}{\partial t^{2}}=2 \frac{\bar{U}}{c^{2}} \frac{\partial^{2} p}{\partial z \partial t}+\frac{\bar{U}^{2}}{c^{2}} \frac{\partial^{2} p}{\partial z^{2}}-2 \bar{\rho} \frac{\operatorname{grad}_{2}}{\bar{U}} \frac{\partial u_{r}}{\partial z}+\frac{1}{\bar{\rho}} \overrightarrow{\operatorname{grad}}_{2} \bar{\rho} \frac{\partial p}{\partial r}
$$

où $\mathrm{u}_{\mathrm{r}}$ est la composante de la vitesse acoustique suivant $\overrightarrow{\mathrm{r}}$ et $\overrightarrow{\operatorname{grad}}_{2}$ défini par :

$$
\overrightarrow{\operatorname{grad}}_{2}=\left(\frac{\partial}{\partial \mathrm{r}} ; \frac{\partial}{\mathrm{r} \partial \theta}\right)
$$

Les trois premiers termes du membre de droite symbolisent les effets de l'écoulement moyen sur la propagation. Le dernier terme exprime quant à lui le fait que l'onde se développe dans un milieu nonisotherme, indiquant ainsi l'influence du champ de temperature non-uniforme sur la pression acoustique.

L'équation (4) peut être écrite sous forme adimensionnelle en considérant $L, R$ et $\tau$ comme longueur, rayon et temps de référence, $\mathrm{v}_{0}, \mathrm{U}_{0}$ et $\mathrm{c}_{0}$ comme vitesse acoustique, vitesse de débit et célérité de réfence (avec $\mathrm{L}=\mathrm{c}_{0} . \tau$ ), et $\rho_{0}$ comme masse volumique de référence :

(5) $\frac{L^{2}}{R^{2}} \Delta_{2} p+\left(1-M_{0}^{2} \frac{\bar{U}^{2}}{c^{2}}\right) \frac{\partial^{2} p}{\partial z^{2}}-\frac{1}{c^{2}} \frac{\partial^{2} p}{\partial t^{2}}=2 M_{0} \frac{\bar{U}}{c} \frac{\partial^{2} p}{\partial z \partial t}-2 \bar{\rho} M_{0} \overrightarrow{g r a d}_{2} \bar{U} \frac{\partial u_{r}}{\partial z}+\frac{1}{\bar{\rho}} \frac{L_{R}^{2}}{R^{2}} \overrightarrow{\operatorname{grad}}_{2} \frac{\partial p}{\partial r}$ où $\mathrm{M}_{0}$ est le nombre de $\mathrm{Mach}, \mathrm{M}_{0}=U \mathrm{U} / \mathrm{c}_{0}$, et $\mathrm{U}_{0}$ la vitesse de débit définie par :

$$
\mathrm{U}_{0}=\frac{1}{\mathrm{~S}} \int_{S} \overline{\mathrm{U}}(\mathrm{r}, \theta) \mathrm{ds}
$$

\section{Oscillations harmoniques}

On examine ici la propagation d'ondes acoustiques harmoniques dans un conduit cylindrique circulaire à paroi rigide. Les pression et vitesse acoustiques adimensionnelles peuvent donc s'écrire sous la forme suivante :

$$
p(r, \theta, z, t)=\operatorname{Re}\left[P(r, \theta) e^{2 \pi j\left(t-\frac{z}{a c_{\varphi}}\right)}\right] ; \quad u_{r}(r, \theta, z, t)=\operatorname{Re}\left[U_{r}(r, \theta) e^{2 \pi j\left(t-\frac{z}{a c_{\varphi}}\right)}\right]
$$

où a vaut 1 pour les ondes se propageant dans le sens de l'écoulement (propagation aval) et -1 pour les ondes se propageant dans le sens inverse de l'écoulement (propagation amont), et où $c_{\varphi}$ est la vitesse de phase adimensionnelle. 
L'équation du mouvement dans la direction radiale permet d'obtenir une relation élémentaire entre les fonctions $P(r, \theta)$ et $U_{r}(r, \theta)$. Finalement, on obtient pour la pression acoustique les équations différentielles suivantes, en posant $P(r, \theta)=P_{r}(r) \cdot P_{\theta}(\theta)$, et en limitant la dépendance des champs de vitesse et de température moyennes à la seule variable $r$ :

$$
\frac{\partial^{2} P_{\theta}}{\partial \theta^{2}}+m^{2} P_{\theta}=0 \text { où m est un entier, } P_{\theta} \text { étant continue en } \theta=0=2 \pi ;
$$

$$
\frac{\partial^{2} P_{r}}{\partial r^{2}}+\left(\frac{1}{r}+f(r)\right) \frac{\partial P_{r}}{\partial r}+\left((k R)^{2} g(r)-\frac{m^{2}}{r^{2}}\right) P_{r}=0
$$

avec

$$
f(r)=\frac{-2 M_{o}}{M_{o} \bar{U}-a c_{\varphi}} \frac{\partial \bar{U}}{\partial r}-\frac{1}{\bar{\rho}} \frac{\partial \bar{p}}{\partial r} \text { et } g(r)=\frac{1}{c^{2}}-\frac{1}{c_{\varphi}^{2}}+\frac{M_{o}}{c_{\varphi}} \frac{\bar{U}}{c}\left(\frac{M_{o}}{c_{\varphi}} \frac{\bar{U}}{c}-2 a\right)
$$

Sur la paroi rigide de la conduite, la vitesse acoustique normale doit rester nulle. Ceci impose la condition aux limites suivante $: \partial P(r, \theta) / \partial r=0$ pour $r= \pm R$. En fait, pour une configuration cylindrique circulaire, ceci revient à considérer deux types de conditions aux limites différentes $: \partial \mathrm{P}_{\mathrm{r}}(\mathrm{r}) / \partial \mathrm{r}=0$ en $\mathrm{r}$ $=0$ et $r=R$ pour les modes circulaires axisymétriques (c.a.d. indépendants de $\theta$, soit $m=0$ ), et $P_{r}(r)=0$ en $\mathrm{r}=0$ et $\partial \mathrm{P}_{\mathrm{r}}(\mathrm{r}) / \partial \mathrm{r}=0$ en $\mathrm{r}=\mathrm{R}$ pour les modes circulaires non-axisymétriques (c.a.d. dépendants de $\theta$, soit $\mathrm{m}>0$ ).

\section{VITESSE DE PHASE}

La présence d'un écoulement non-uniforme et non-isotherme a en particulier pour effet de modifier la vitesse de phase des modes propagatifs. La vitesse de phase du mode longitudinal $(m=0)$ peut être obtenue à partir d'une méthode de perturbation tant que le nombre de Mach et les écarts de température restent faibles. En intégrant la relation (7) sur la section $S$ du conduit, on obtient, compte tenu du théorème de Green généralisé :

$$
\text { (8) } \int_{S}\left(\frac{1}{r}+f(r)\right) \frac{\partial P_{r}}{\partial r} d s+\int_{S}(k R)^{2} g(r) P_{r} d s=0
$$

Les développements en série du nombre de Mach $\mathrm{M}_{0}$ et du paramètre $\varepsilon$, représentatif des écarts de température, s'écrivent pour la pression et la vitesse de phase :

$$
\begin{aligned}
& P_{r}(r)=P_{0}+\varepsilon P_{10}+M_{o} P_{01}+\ldots \\
& c_{\varphi}=c_{\varphi_{0}}\left(1+\varepsilon \gamma_{10}+M_{0} \gamma_{01}+\ldots\right) \quad \text { avec } \varepsilon=\frac{T_{1}-T_{0}}{T_{0}} \quad(\varepsilon \ll 1)
\end{aligned}
$$

En première approximation (à l'ordre 0 ), on peut admettre que $\mathrm{P}_{\mathrm{r}}(\mathrm{r})=\mathrm{P}_{0}$, en considérant que la répartition du mode longitudinal n'est pas modifiée par la présence de l'écoulement et du gradient de température. Il est alors possible d'obtenir les termes du premier ordre pour la vitesse de phase $c_{\varphi}$, l'expression (8) se réduisant à la forme suivante :

$$
\text { (9) } \int_{S}\left(\frac{1}{c^{2}}-\frac{1}{c_{\varphi}^{2}}-\frac{2 a M_{o} \bar{U}}{c_{\varphi} c}\right) d s=0 \text {. }
$$

En posant $c^{2}=1+\varepsilon h(r)$, où $h(r)=\frac{T(r)-T_{0}}{T_{1}-T_{0}}$, on obtient :

- à l'ordre $0: c_{\varphi 0}=1$;

- à l'ordre 1 en $\varepsilon: \gamma_{10}=\frac{1}{2} \frac{T_{m}-T_{0}}{T_{1}-T_{0}} \quad$ où $\quad T_{m}=\frac{1}{S} \int_{S} \vec{T}(r) d s ;$

- à l'ordre 1 en $\mathrm{M}_{0}: \gamma_{01}=\mathrm{a}$. 
La vitesse de phase adimensionnelle du mode longitudinal s'ecrit donc :

(10) $c_{\varphi}=1+\frac{1}{2} \frac{T_{m}-T_{0}}{T_{0}}+a \cdot M_{0}$

\section{RESOLUTION ET DISCUSSION}

La détermination de la répartition de la pression acoustique sur un rayon du conduit est effectuée à partir de l'équation (7), résolue par la méthode numérique de Runge-Kutta à l'ordre 4. On suppose une répartition linéaire de la temperature, de telle sorte que $T(r)=1+r\left(T_{1}-T_{0}\right) / T_{0}$. De même, on considère un ecoulement laminaire tel que $U(r)=2\left(1-r^{2}\right)$. Les temperatures $T_{1}$ et $T_{0}$ sont respectivement prises égales $\grave{a} 400 \mathrm{~K}$ et $300 \mathrm{~K}$. On considère plusieurs valeurs du nombre de Mach $\left(\mathrm{M}_{0}=0 ; 0,1 ; 0,2 ; 0,4\right)$ afin d'examiner l'influence du gradient de vitesse. Les calculs sont conduits pour les propagations aval et amont.

On détermine tout d'abord les fréquences de coupure de chaque mode propagatif. Celles-ci sont obtenues à partir de l'équation (7) dans laquelle on fait tendre la vitesse de phase vers l'infini, ce qui revient à considérer que l'ensemble du fluide vibre en phase dans les directions radiale ou azimutale. Les valeurs obtenues sont indépendantes du gradient de vitesse et du type de propagation (aval ou amont), et identiques à celles qu'on obtiendrait dans un milieu isotherme de temperature égale à la température moyenne $T_{m}$ (écart $\neq 1 \%$ ).

La vitesse de phase ainsi que la répartition de pression sont ensuite obtenues à partir des l'equation (7), dans laquelle on fixe la valeur du nombre d'onde adimensionnel $\mathrm{kR}$. On remarque que la vitesse de phase du mode considéré est liée à la fois au gradient de vitesse et au gradient de temperature, et selon qu'on s'intéresse à la propagation amont ou aval. Les figures 1 et 2 montrent l'évolution de la pression acoustique du mode fondamental et du mode circulaire d'ordre 1 pour une valeur donnée de $\mathrm{kR}$ $(k R=5)$, en propagation aval et amont. Les gradients influencent fortement sur la répartition de pression, mais agissent différamment. Le gradient de température implique de plus fortes oscillations de la pression acoustique dans les zones les plus froides, et ceci indépendamment du type de propagation (aval ou amont). La réfraction des ondes qui résulte du gradient de vitesse est quant à elle différente selon le type de propagation. La pression acoustique est concentrée dans les zones de forts gradients de vitesse (près des parois) en propagation aval, et dans les zones de faibles gradients (près du centre) en propagation amont. L'ensemble indique donc que la propagation des ondes est très perturbée en milieu inhomogène, même pour des variations relativement faibles des champs moyens.
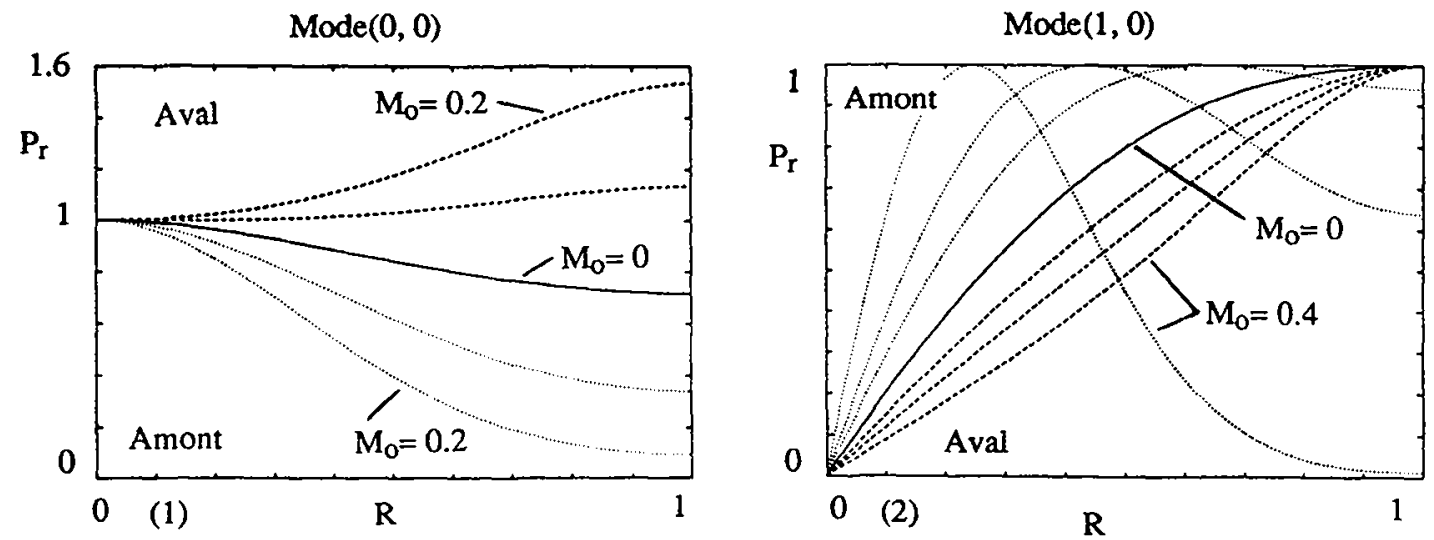

Fig. 1 et 2 - Répartition de la pression du mode longitudinal (1) et du mode circulaire d'ordre $1(2)$ pour $\mathrm{kR}=5$. Propagations aval et amont $\left(\mathrm{M}_{0}=0 ; 0,1 ; 0,2 ; 0,4\right)$.

[1] PRIDMORE-BROWN, J. Fluid Mech., 4, (1958), 393-406.

[2] MOHRING, MULLER and OBERMEIER, Rev, Modern Physics, 55, (1983), 707-724.

[3] CAMPOS, Rev. d'Acoustique, 67, (1983), 217-233.

[4] GERVAIS and PEUBE, Inter-Noise90, (1990), 567-570. 